\title{
DOA Estimation for UCAs Based on Virtual Subarray Beamforming Technique
}

\author{
Bo Xu${ }^{1}$, Zhigang Huang ${ }^{1, \text { a }}$ \\ ${ }^{1}$ Key Laborartory of Science and Technology for National Defence on Blind Signal Processing, 612500 Chengdu, China
}

\begin{abstract}
Direction-of-arrival (DOA) estimation is always a hotspot research in the fields of radar, sonar, communication and so on. And uniform circular arrays (UCAs) are more attractive in the context of DOA estimation since their symmetrical structures have potential to provide two directions coverage. This paper proposed a new DOA estimation method for UCAs via virtual subarray beamforming technique. The method would provide an acceptable DOA estimate even if the number of sources is great than the number of array elements. Also, the performance of the proposed method would hold good when the snapshot length or the signal-to-noise ratio (SNR) is small. Simulations show that the proposed technique offers significantly improved estimation resolution, capacity, and accuracy relative to the existing techniques.
\end{abstract}

\section{INTRODUCTION}

Direction-of-arrival (DOA) usually plays a very important role in many applications, such as radar, sonar and wireless communication systems. The uniform circular array (UCA) is more attractive than the uniform linear array (ULA) in the context of DOA estimation since it would provide two directions coverage, a $90^{\circ}$ elevation angle and full $360^{\circ}$ azimuth angle. Many DOA estimation algorithms for UCAs have been proposed, like UCA-RB-MUSIC, UCA-ESPRIT and so on [1-6]. However, these meth-ods provide an acceptable DOA estimate if the num-ber of sources is less than the number of array ele-ments [7]. On the other hand, the performances of these methods suffer considerable degradation when the snapshot length or the signal-tonoise ratio (SNR) is small [8].

Wang have proposed a worth watching technique for DOA estimation, called SBDOA algorithm [9]. In this algorithm, two subarrays are used to form a sig-nal whose phase relative to the reference signal is a function of the DOA. The DOA is then estimated based on the computation of the phase shift between the reference signal and its phase-shifted version. As the author pointed out, since the phase-shifted refer-ence signal is obtained after interference rejection through beamforming, the effect of cochannel inter-ference on the estimation is significantly reduced. More important, this algorithm would provide a fa-vorable performance even if the number of sources is great than the number of array elements. However, it is regrettable that the SBDOA technique is derived from uniform linear arrays (ULAs), and would not be employed by UCAs directly, which is more attractive in some applications like satellite and mobile com-munications.
This paper can be considered as an extension of the research in [9], where we spread the SBDOA technique to UCAs, named UCA-VSBDOA algorithm. The letters "VSB" mean "Virtual Subarray Beamforming". Because in our method, the two subarrays are composed two virtual subarrays. But the term of Bessel function in the response vector of VULA caused difficulties to derive the UCA-VSBDOA estimator. Thus, we introduced a weighting matrix to solve this problem. Also, the LMS algorithm has been employed to avoid the matrix inversion since the signal correlation matrix may be illconditioned. Simulations proved the correction of the proposed technique.

\section{PROBLEM FORMULATION}

\subsection{ULA-SBDOA Algorithm}

See from Fig.1, an N-elements ULA with inter-element spacing $\mathrm{r}$ deploys at a receiver. $\mathrm{K}$ uncorrelat-ed sources and $\mathrm{H}$ unknown interferences impinge on the array from the far field. The down-converted receiving signal at the array can be represented as

$$
u_{n}(t)=\sum_{k=1}^{K+H} s_{k}(t)\left[e^{-j k_{0} r \sin \left(\theta_{k}\right)}\right]^{n-1}+w_{n}(t), n=1,2, \cdots, N(1)
$$

Where, $s_{k}(t)$ for $k=1,2, \ldots K$ is a target source component, and $s_{k}(t)$ for $k=K+1, K+2, \ldots K+H$ is an unknown interference component. $k_{0}=2 \pi / \lambda$ is the wavenumber, and $\lambda$ is the carrier wavelength. $\theta_{k}$ is the DOA of the $k$ th target. $w_{n}(t)$ is the background Gauss noise of the $n$th element. The two maximum overlap subarrays (MOSs), which is introduced in [9][11,12], would be defined as

$$
\mathbf{y}_{\mathrm{A}}(t)=\left[\begin{array}{llll}
u_{1}(t) & u_{2}(t) & \cdots & u_{N-1}(t)
\end{array}\right]^{T}
$$

\footnotetext{
*Corresponding author: ${ }^{\mathrm{a}}$ h-zhigang@163.com
} 


$$
\mathbf{y}_{\mathrm{B}}(t)=\left[\begin{array}{llll}
u_{2}(t) & u_{3}(t) & \cdots & u_{N}(t)
\end{array}\right]^{T}
$$

Obviously, there would be

$$
\mathbf{y}_{\mathrm{B}}(t)=e^{-j k_{0} r \sin \left(\theta_{k}\right)} \mathbf{y}_{\mathrm{A}}(t)
$$

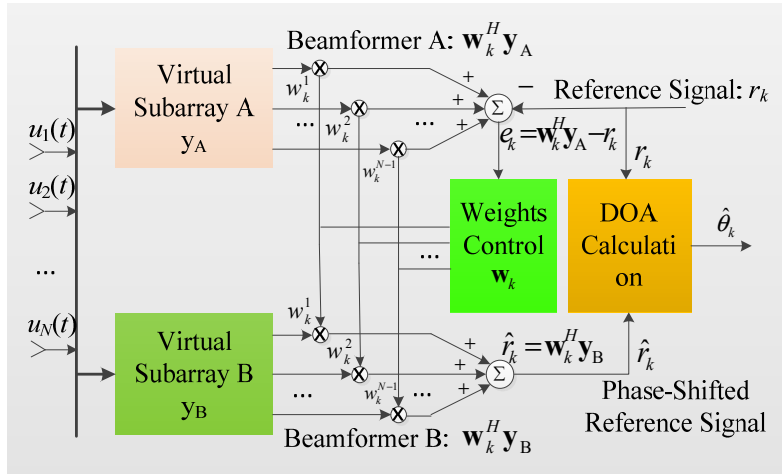

Figure 1. The block diagram of SBDOA algorithm [9]

Then the phase shift of the $k$ th source between $\mathbf{y}_{\mathrm{A}}(t)$ and $\mathbf{y}_{\mathrm{B}}(t)$ would be

$$
\varphi_{k}=-k_{0} r \sin \left(\theta_{k}\right)
$$

As Fig.1 shows, two beamformers are constructed to obtain the phase-shifted reference signal whose phase relative to the target DOA. The weight vectors of the two beamformers can be described as two optimum problems in the condition of MMSE sense

$$
\begin{array}{ll}
\min _{k}^{\mathrm{A}} & E\left[\left|\left(\mathbf{w}_{k}^{\mathrm{A}}\right)^{H} \mathbf{y}_{\mathrm{A}}(t)-r_{k}(t)\right|^{2}\right] \\
\min _{\mathbf{w}_{k}^{\mathrm{B}}} & E\left[\left|\left(\mathbf{w}_{k}^{\mathrm{B}}\right)^{H} \mathbf{y}_{\mathrm{B}}(t)-e^{j \varphi_{k}} r_{k}(t)\right|^{2}\right]
\end{array}
$$

Where, $r_{k}$ is the $k$ th reference signal. Here, the reference signal is a pilot signal (or decision-directed signal) which is common in many applications [10]. The optimal weight vectors $\mathbf{w}_{k}^{\mathrm{A}}$ and $\mathbf{w}_{k}^{\mathrm{B}}$ can be readily obtained in closed form as

$$
\begin{aligned}
\mathbf{w}_{k}^{A} & =\mathbf{R}_{A}^{-1} \mathbf{h}_{k}^{A} \\
\mathbf{w}_{k}^{B} & =\mathbf{R}_{B}^{-1} \mathbf{h}_{k}^{B}
\end{aligned}
$$

Where,

$$
\begin{aligned}
& \left\{\begin{array}{l}
\mathbf{R}_{\mathrm{A}}=E\left[\mathbf{y}_{\mathrm{A}}(t) \mathbf{y}_{\mathrm{A}}^{H}(t)\right] \\
\mathbf{h}_{k}^{\mathrm{A}}=E\left\{\left[r_{k}(t)\right]^{*} \mathbf{y}_{\mathrm{A}}(t)\right\}
\end{array}\right. \\
& \left\{\begin{array}{l}
\mathbf{R}_{\mathrm{B}}=E\left[\mathbf{y}_{\mathrm{B}}(t) \mathbf{y}_{\mathrm{B}}^{H}(t)\right] \\
\mathbf{h}_{k}^{\mathrm{B}}=E\left\{e^{-j \varphi_{k}}\left[r_{k}(t)\right]^{*} \mathbf{y}_{\mathrm{B}}(t)\right\}
\end{array}\right.
\end{aligned}
$$

Since $\varphi_{k}$ is unknown, $\mathbf{w}_{k}^{\mathrm{B}}$ would not be determined by (7) directly. However, theoretic analysis shows that $\mathbf{w}_{k}^{\mathrm{B}}=\mathbf{w}_{k}^{\mathrm{A}}$ (see [9] for more details). So the phase shifted reference signal can be estimated using $\mathbf{w}_{k}^{\mathrm{A}}$ in (6) feasibly. If

$$
\hat{r}_{k}(t)=\left(\mathbf{w}_{k}^{\mathrm{A}}\right)^{H} \mathbf{y}_{\mathrm{B}}(t)
$$

denotes the phase-shifted reference signal estimated by Beamformer $\mathrm{B}$, then the phase shift of the $k$ th source between $\mathbf{y}_{\mathrm{A}}(t)$ and $\mathbf{y}_{\mathrm{B}}(t)$ would be determined as

$$
\varphi_{k}=k_{0} r \sin \left(\theta_{k}\right)=\arg \left(\mathbf{r}_{k}^{H} \hat{\mathbf{r}}_{k}\right)
$$

Where, $\mathbf{r}_{k}=\left[r_{k}(1) r_{k}(2) \cdots r_{k}(L)\right]^{T}, \hat{\mathbf{r}}_{k}=\left[\hat{r}_{k}(1) \hat{r}_{k}(2) \cdots \hat{r}_{k}(L)\right]^{T}$, and $L$ denotes the snapshot length. The target DOA of the $k$ th source would be

$$
\hat{\theta}_{k}=\arcsin \left[-\arg \left(\mathbf{r}_{k}^{H} \hat{\mathbf{r}}_{k}\right) / k_{0} r\right]
$$

\subsection{Problem Description}

There are some impracticable troubles while spreading the ULA-SBDOA algorithm to UCAs directly. The first is that the element in response vector of UCA is not geometric, which goes against the generation of subarrays. Though, one would use the mode excitation method in [13] to transform the UCA in element space into a VULA in phase mode space ideally. But the Bessel function with different order is introduced into the response vector of VULA by the referred transformation, which caused the fact that $\mathbf{w}_{k}^{\mathrm{B}} \neq \mathbf{w}_{k}^{\mathrm{A}}$. So the phase-shifted reference signal would not be estimated directly. Otherwise, it is difficult to determine the inversion of $\mathbf{R}_{\mathrm{A}}$ directly sometimes, since $\mathbf{R}_{\mathrm{A}}$ may be ill-conditioned while a few sources (compared to the number of array elements) impinge on the array with high SNR.

\section{PROBLEM SOLUTION AND UCA- VSBDOA ALGORITHM}

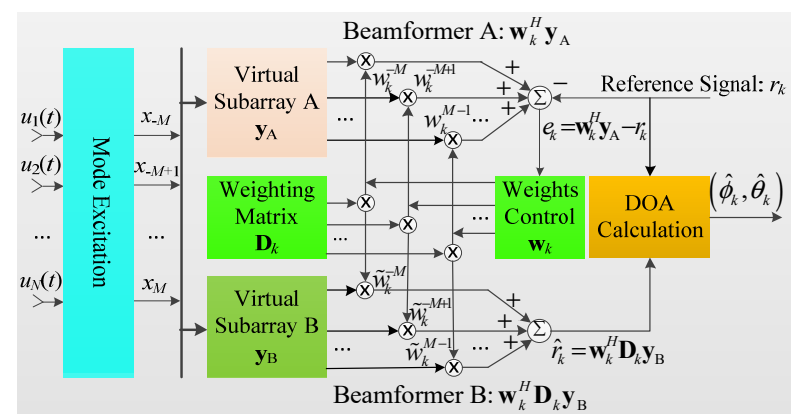

Figure 2. The block diagram of UCA-VSBDOA algorithm

\subsection{Mode excitation}

The block diagram of UCA-VSBDOA algorithm is shown in Fig.2. As mentioned before, we first introduced the mode excitation method in [13] to solve the first problem. Consider $K$ uncorrelated sources and $H$ unknown interferences impinge on an $\mathrm{N}$-elements and $r$ radius UCA from the far field. The signal of the $m$ th virtual element in phase mode space after mode excitation would be [13]

$$
x_{m}(t)=\sum_{k=1}^{K+H} J_{m}\left(\varsigma_{k}\right) e^{j m \theta_{k}} s_{k}(t), m=-M, \ldots, M
$$

Where, $J_{m}\left(\varsigma_{k}\right)$ is the Bessel function of the first kind of order $m$, and $\varsigma_{k}=k_{0} r \sin \left(\phi_{k}\right) .\left(\phi_{k}, \theta_{k}\right)$ denotes the elevation and azimuth angles respectively. $M$ denotes the mode order which equals to floor $\left(k_{0} r\right)$ [13]. In this situation, the two MOSs can be described as 
Where,

$$
\begin{gathered}
\mathbf{J}_{\mathrm{A}}=\operatorname{diag}\left[J_{-M}\left(\varsigma_{k}\right), J_{-M+1}\left(\varsigma_{k}\right), \ldots, J_{M-1}\left(\varsigma_{k}\right)\right] \\
\mathbf{J}_{\mathrm{B}}=\operatorname{diag}\left[J_{-M+1}\left(\varsigma_{k}\right), J_{-M+2}\left(\varsigma_{k}\right), \ldots, J_{M}\left(\varsigma_{k}\right)\right] \\
\mathbf{A}_{\mathrm{VULA}}=\left[e^{-j M \theta_{k}}, e^{-j(M-1) \theta_{k}}, \ldots, e^{j(M-1) \theta_{k}}\right]^{T} \\
\mathbf{B}_{\mathrm{VULA}}=\left[e^{-j(M-1) \theta_{k}}, e^{-j(M-2) \theta_{k}}, \ldots, e^{j M \theta_{k}}\right]^{T}
\end{gathered}
$$

Thus, there is

$$
\left\{\begin{aligned}
\mathbf{J}_{\mathrm{A}} & \neq \mathbf{J}_{\mathrm{B}} \\
\mathbf{A}_{\mathrm{VULA}} & =e^{j \theta_{k}} \mathbf{B}_{\mathrm{VULA}}
\end{aligned}\right.
$$

\subsection{Weighting matrix}

As shown in Fig.2, two beamformers are used to estimate the weight vector and the phase-shifted reference signal respectively while determining the DOA of the kth target. But by substituting (16) and (17) into (8) and (9), we have

$$
\begin{aligned}
& \mathbf{w}_{k}^{\mathrm{A}}=\sum_{k=1}^{K+H} \sigma_{k}^{-1} \sigma_{r} \mathbf{J}_{\mathrm{A}}^{-1}\left(\mathbf{A}_{\mathrm{VULA}} \cdot \mathbf{A}_{\mathrm{VULA}}^{H}\right)^{-1} \mathbf{A}_{\mathrm{VULA}} \\
& \mathbf{w}_{k}^{\mathrm{B}}=\sum_{k=1}^{K+H} \sigma_{k}^{-1} \sigma_{r} \mathbf{J}_{\mathrm{B}}^{-1}\left(\mathbf{A}_{\mathrm{VULA}} \cdot \mathbf{A}_{\mathrm{VULA}}^{H}\right)^{-1} \mathbf{A}_{\mathrm{VULA}}
\end{aligned}
$$

Where, $\sigma_{k}^{2}=\left|s_{k}\right|^{2}$ and $\sigma_{r}^{2}=\left|r_{k}\right|^{2}$ are power of the received signal and the reference signal respectively. Obviously, there is $\mathbf{w}_{k}^{\mathrm{B}} \neq \mathbf{w}_{k}^{\mathrm{A}}$ because $\mathbf{J}_{\mathrm{A}} \neq \mathbf{J}_{\mathrm{B}}$, which caused difficulties to estimate the phase-shifted reference signal.

In this paper, we introduced a weighting matrix to solve this problem. Let

$$
\mathbf{D}_{k}=\operatorname{diag}\left[\frac{J_{-M}\left(\varsigma_{k}\right)}{J_{-M+1}\left(\varsigma_{k}\right)}, \frac{J_{-M+1}\left(\varsigma_{k}\right)}{J_{-M+2}\left(\varsigma_{k}\right)}, \ldots, \frac{J_{M-1}\left(\varsigma_{k}\right)}{J_{M}\left(\varsigma_{k}\right)}\right]
$$

denote the weighting matrix. Obviously, to keep the weighting matrix valid, the denominator in (25) should avoid becoming zero. Actually, the visible region $\phi_{k} \in[0, \pi / 2]$ translates into $\varsigma_{k} \in\left[0, k_{0} r\right]$. See from Fig.3, the first zero crossing point of the first kind Bessel function is $J_{0}\left(\varsigma_{\text {zero }}\right)$ and $\zeta_{\text {zero }} \approx 2.4$ theoretically. Thus, if $\phi_{k} \neq 0$ and $k_{0} r<\varsigma_{\text {zero }}, J_{m}\left(\varsigma_{k}\right)$ would be nonzero. A typical value is $r=0.4 \lambda$. Under this situation, there would be $\mathbf{J}_{\mathrm{A}}=\mathbf{D}_{k} \mathbf{J}_{\mathrm{B}}$. And if $\varsigma=\varsigma_{k}$, then the optimum estimation of phase-shifted reference signal is

$$
\hat{r}_{k}(t)=\mathbf{w}_{k}^{\mathrm{A}} \mathbf{D}_{k} \mathbf{y}_{\mathrm{B}}(t)=e^{j \theta_{k}} r_{k}(t)+w_{k}(t)
$$

Then the azimuth of the $k$ th target source can be evoked by

$$
\theta_{k}=\arg \left(\mathbf{r}_{k}^{H} \hat{\mathbf{r}}_{k}\right)
$$

Where, $\mathbf{r}_{k}=\left[r_{k}(1) r_{k}(2) \cdots r_{k}(L)\right]^{T}, \hat{\mathbf{r}}_{k}=\left[\hat{r}_{k}(1) \hat{r}_{k}(2) \cdots \hat{r}_{k}(L)\right]^{T}$, and $L$ denotes the snapshot length. Or else, we can search different elevation angles to get the corresponding azimuth angle estimates. The target DOA is the one maximizes the power of array output. It can be described as

$$
\left\{\left(\hat{\phi}_{k}, \hat{\theta}_{k}\right) \mid \max \left[\sum_{l=0}^{L}\left(\sum_{n=1}^{N}\left[u_{n}(t) e^{-j \psi_{k, n}}\right]\right)^{2} / L\right]\right\}
$$

Where, $\left(\hat{\phi}_{k}, \hat{\theta}_{k}\right)$ is the estimated DOA of the $k$ th target source component.

$$
\psi_{k, n}=k_{0} r \sin \left(\hat{\phi}_{k}^{\varsigma}\right) \cos \left[\hat{\theta}_{k}^{\varsigma}-2 \pi(n-1) / N\right]
$$

$\left(\hat{\phi}_{k}^{\varsigma}, \hat{\theta}_{k}^{\varsigma}\right)$ corresponds to the searched elevation angle and the computed azimuth angle.

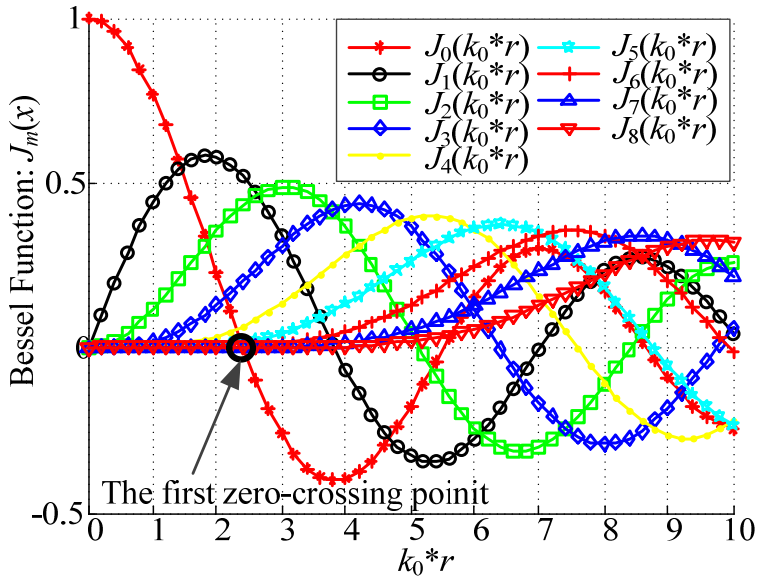

Figure 3. The Bessel function of the first kind of order $m$.

\subsection{Weight Vector Adaptation}

As referred above, the autocorrelation matrix of $\mathbf{y}_{\mathrm{A}}$ may be ill-conditioned. To avoid the matrix inversion, the LMS method in [14] is employed to obtain the estimate of $\mathbf{w}_{k}^{\mathrm{A}}$. If we let

$$
e_{k}(t)=\left(\hat{\mathbf{w}}_{k}^{\mathrm{A}}\right)^{H} \mathbf{y}_{\mathrm{A}}(t)-r_{k}(t)
$$

denote the error function between the reference signal and the output of beamformer A. In mean square error sense, there is

$$
\hat{\nabla}(t)=\frac{\partial e_{k}^{2}(t)}{\partial \hat{\mathbf{w}}_{k}^{\mathrm{A}}(t)}=-2 \mathbf{h}_{k}^{\mathrm{A}}(t)+2 \mathbf{R}_{\mathrm{A}}(t) \hat{\mathbf{w}}_{k}^{\mathrm{A}}(t)
$$

According to LMS algorithm, we have

$$
\hat{\mathbf{w}}_{k}^{\mathrm{A}}(t+1)=\hat{\mathbf{w}}_{k}^{\mathrm{A}}(t)+\mu[-\hat{\nabla}(t)]
$$

Where, $\mu$ is the step size within $\left(0, \operatorname{tr}\left[\mathbf{R}_{\mathrm{A}}\right]^{-1}\right)$. If $\left|e_{k}(t)\right|$ is less than a given threshold, there would be $\mathbf{w}_{k}^{\mathrm{A}} \approx \hat{\mathbf{w}}_{k}^{\mathrm{A}}$.

\subsection{Summarization of UCA-VSBDOA algorithm}

Step1. Utilization of the mode excitation method to transform a UCA into a VULA and generate two MOSs.

Step2. Employ of the LMS algorithm to estimate the weight vector $\hat{\mathbf{w}}_{k}^{\mathrm{A}}$ via (31) and (32) with an appropriate $\mu$.

Step3. Search of the different elevation angles in the visible range to estimate the phase-shifted reference signal using (26). 
Step4. Estimation of the DOA of the $k$ th target source using (27) and (28).

\section{SIMULATIONS}

We mainly compare the proposed UCA-VSBDOA algorithm with the UCA-RB-MUSIC and UCA-ESPRIT from accuracy, resolution, and capability of DOA estimation in terms of root mean square error (RMSE) and success probability, which are defined as

$$
\text { RMSE }= \begin{cases}\sqrt{\frac{1}{N_{\text {sim }}} \sum_{i=1}^{N_{\text {sim }}}\left(\hat{\phi}_{k}-\phi_{k}\right)^{2}} & \text { for elevation angle } \\ \sqrt{\frac{1}{N_{\text {sim }}} \sum_{i=1}^{N_{\text {sim }}}\left(\hat{\theta}_{k}-\theta_{k}\right)^{2}} & \text { for azimuth angle }\end{cases}
$$

Success Probability $=N_{\text {correct }} / N_{\text {sim }}$

Where, $\left(\hat{\phi}_{k}, \hat{\theta}_{k}\right)$ and $\left(\phi_{k}, \theta_{k}\right)$ denote the estimated DOA and the real DOA of the $k$ th target source respectively. $N_{\text {sim }}$ is the total simulation times, and $N_{\text {correct }}$ represents the times that the errors between the estimated DOA and the real DOA are less than $0.5^{\circ}$.

During the simulations, we employed an 8-elements UCA, whose radius $r=0.4 \lambda$ and $\lambda=0.2 \mathrm{~m}$. The noise of each element can be approximated as a white Gauss noise. The different target sources and reference signals are composed by direct spread spectrum signals (DSSS) with different code offsets, where the code length and code rate are 1023 and $1.023 \mathrm{MHz}$ respectively. One thousand Mont Carlo simulation runs are performed to evaluate the RMSE or success probability in each experiment. Note that the compared two methods, though, do not need any reference signal in theory. However, DSSS as the pilot or reference signal is common in our daily life such as the third generation mobile communication.

The first simulation deals with a case that only one signal component impinges on the array from $(\phi$, $\theta)=\left(20^{\circ}, 20^{\circ}\right)$. The performances of DOA estimation by UCA-RB-MUSIC, UCA-ESPRIT and the proposed method are depicted as functions of the snapshot length and SNR respectively. Fig.4 shows the convergence rate and mean square error of the LMS algorithm under different $\mu$. It is obvious that $\mu=0.1 / \operatorname{tr}\left[\mathbf{R}_{\mathrm{A}}\right]$ is more suitable since its convergence rate and MSE are moderate. From Fig.5 and Fig.6, we can see that the RMSE of DOA estimation achieved by the proposed method is lower than that of UCA-RB-MUSIC and UCA-ESPRIT, even if the snapshot length or the SNR is small. On the other hand, if $\mu$ is a biggish value such as $\mu=0.9 / \operatorname{tr}\left[\mathbf{R}_{\mathrm{A}}\right]$, the performance of the proposed method does not appear to be much different from that of UCARB-MUSIC. But the accuracy does not improve obviously with decreasing $\mu$. So we would employ $\mu=0.1 / \operatorname{tr}\left[\mathbf{R}_{\mathrm{A}}\right]$ in the other simulations.

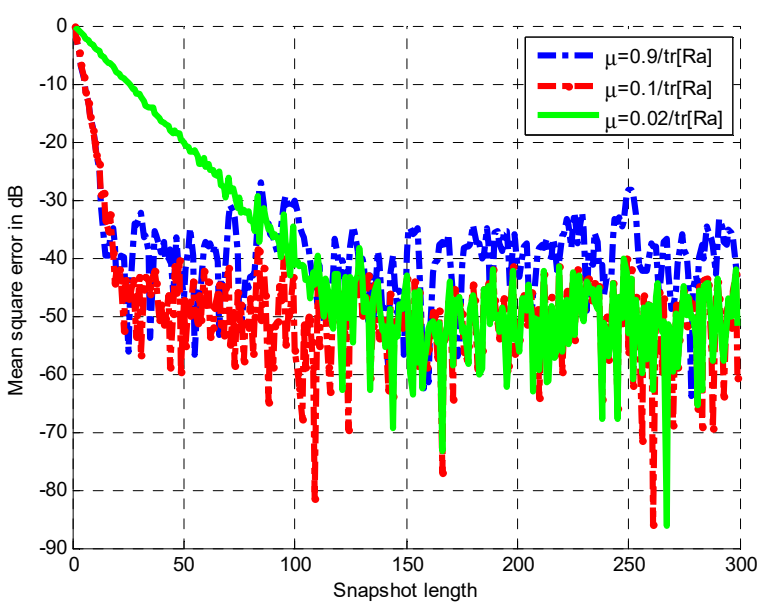

Figure 4. Rate of convergence with different $\mu$ : the $\mathrm{SNR}=10 \mathrm{~dB}$.

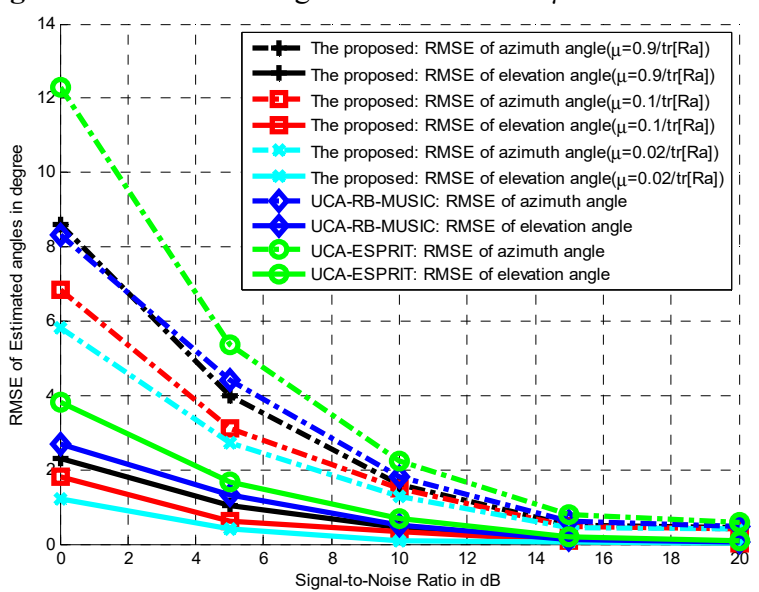

Figure 5. The RMSE versus SNR: the snapshot length is 200.

In the second simulation, we consider the resolution of DOA estimation using different techniques in aspects of elevation angle and azimuth angle respectively. We first assume that there are two target sources impinging on the array from $(\phi, \theta)=\left(20^{\circ}, 20^{\circ}\right)$. One of them is fixed, and the other is transformable in direction of elevation and azimuth angles with a step of $1^{\circ}$. Fig. 7 shows that the success probability of resolution at different angle separations. It can be seen from Fig.7 that our method has a better resolution than the referred methods.

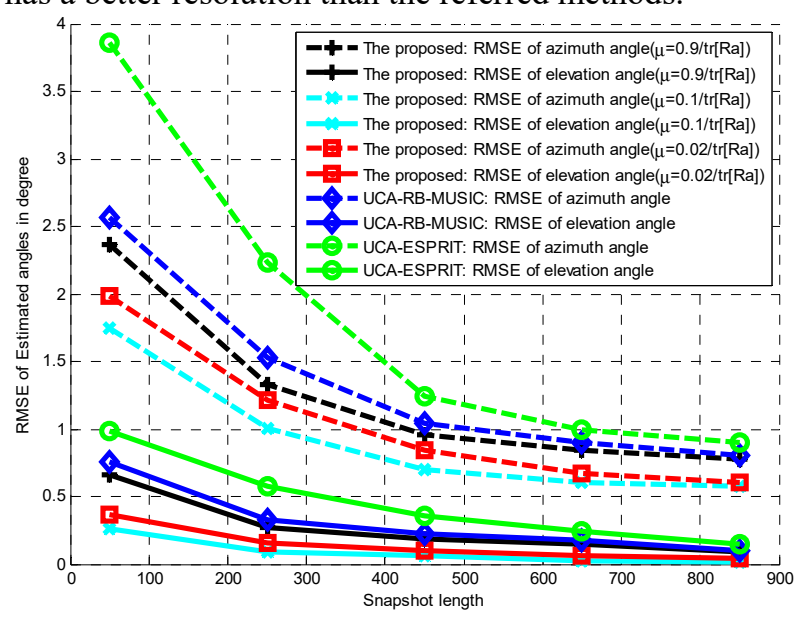

Figure 6. The RMSE versus snapshot length: the $\mathrm{SNR}=10 \mathrm{~dB}$. 


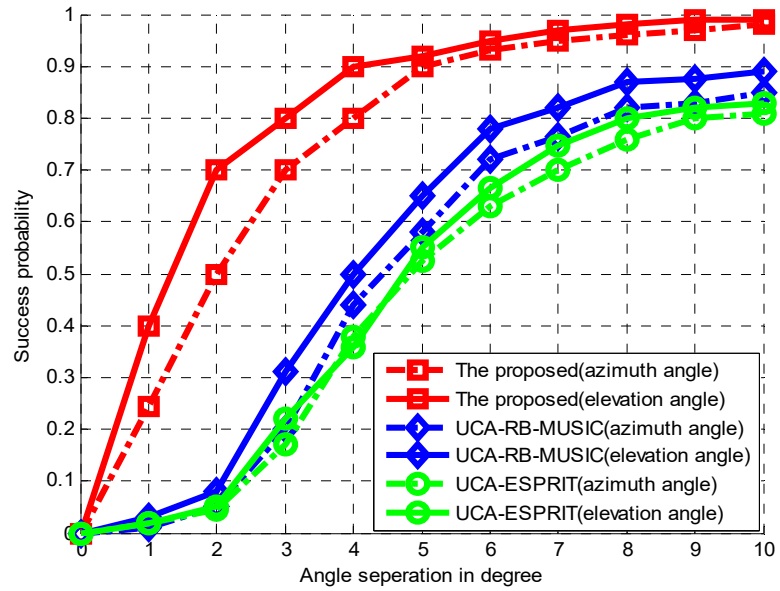

Figure 7.The success probability of angle resolution, where the $\mathrm{SNR}=10 \mathrm{~dB}$ and the snapshot length is 200 .

In the third simulation, the number of sources varied from 1 to 10 with the same power. All the sources are DSS signals coded by different pseudorandom codes to keep them uncorrelated. The DOA of one known target is fixed at $(\phi, \theta)=\left(20^{\circ}, 20^{\circ}\right)$ and the other unknown sources are randomly distributed in the visible range of the array. This simulation is helpful to analyze the capability of DOA estimation. Fig.8 shows the simulation results, where we can see that the RMSE of the proposed method is the lowest in the referred three methods obviously. Especially, the proposed method holds good when the number of sources is bigger than the number of array elements. That means the proposed UCA-VSBDOA method has a better capability of DOA estimation. On the other hand, if the random sources are looked as interferences, this simulation also indicates the proposed method has a good performance on interference rejection.

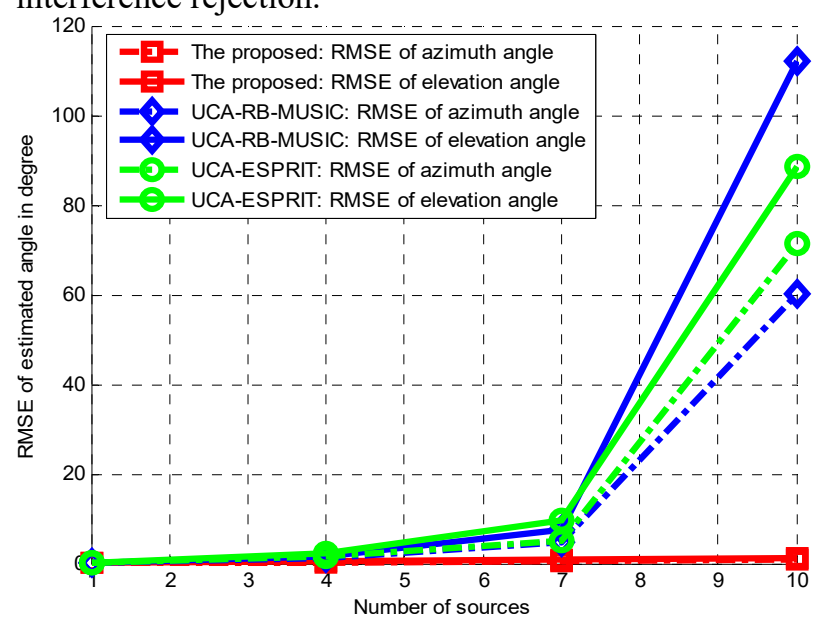

Figure 8. RMSE of the estimated DOA under different number of signal sources, where the $\mathrm{SNR}=10 \mathrm{~dB}$ and the snapshot length is 200 .

\section{CONCLUSION}

A DOA estimation algorithm for UCAs has been proposed in this paper, which can be considered as an extension of the ULA-SBDOA method. In this algorithm, the mode excitation method is employed to transform a UCA in element space into a VULA in phase mode space first. At the same time, two MOSs and beamformers are formed. Since the reference signal is used in the beamformer the weights can be obtained by LMS algorithm. And a weighting matrix is introduced to revise the estimated weight vector which is adopted by the second beamformer to estimate the phase-shifted reference signal. Finally, the target DOA is estimated based on the computation of the phase shift between the reference signal and its phase-shifted version. The proposed scheme has been tested by numeric simulations. Experimental results show that the proposed scheme could provide higher resolution, accuracy and capability of DOA estimation than the referred techniques.

\section{REFERENCES}

1. V. S. Kedia, B. Chandna, SP. 60 (1997)

2. N. Yuen, B. Friedlander, IEEE Trans. On SP. 45 (1997)

3. M. A. Hasan, SP. 77 (1999)

4. M. B. Qi, C. C. Ko, J. Z. Wan, IEEE Trans. On Aero. Elec. Sys. 41 (2005)

5. X. Mestre, M. Á. Lagunas, IEEE Trans. On SP. 56 (2008)

6. P. Palanisamy, N. Kalyanasundaram, A. Raghunandan, SP. 89 (2009)

7. C. T. Chiang, A. C. Chang, IEEE Trans. On Ant. Prop. 51 (2003)

8. C. Qian, L. Huang, H. C. So, IEEE SPL. 21 (2014)

9. N. Y. Wang, P. Agathoklis, A. Antoniou, IEEE Trans. On SP. 54 (2006)

10. S. Tanaka, M. Sawahashi, F. Adachi, IEICE. Trans. On Fun. 12 (1997)

11. V. C. Soon, Y. F. Huang, IEEE. Trans. On SP. 40 (1992)

12. N. Tayem, H. M. Kwon, IEEE Trans. On Ant. Prop. 52 (2004)

13. C. P. Mathews, M. D. Zoltowski, IEEE. Trans. On SP. 9 (1994)

14. S. Lim, J. Lee, J. Park, IEEE Veh. Technol. Conf. 4 (2007)

15. M. Wax, T. Kailath, IEEE Trans. On ASSP. 33 (1985)

16. Y. J. Gu, N. A. Goodman, S. H. Hong, Y. Li, SP. 96 (2014) 